\title{
Perceptions of portion size and energy content: implications for strategies to affect behaviour change
}

\author{
Emily Brindal ${ }^{1, *}$, Carlene Wilson ${ }^{2}$, Philip Mohr ${ }^{1}$ and Gary Wittert ${ }^{3}$ \\ ${ }^{1}$ CSIRO Food and Nutritional Sciences - Adelaide, PO Box 10041, Adelaide BC, South Australia 5000, \\ Australia: ${ }^{2}$ Cancer Council South Australia and Flinders Centre for Cancer Prevention and Control, \\ School of Medicine, Flinders University of South Australia, Adelaide, Australia: ${ }^{3}$ Discipline of Medicine, \\ University of Adelaide, Adelaide, Australia
}

Submitted 6 September 2010: Accepted 2 May 2011: First published online 23 June 2011

\begin{abstract}
Objective: To assess Australian consumers' perception of portion size of fast-food items and their ability to estimate energy content.

Design: Cross-sectional computer-based survey.

Setting: Australia.

Subjects: Fast-food consumers (168 male, 324 female) were asked to recall the items eaten at the most recent visit to a fast-food restaurant, rate the prospective satiety and estimate the energy content of seven fast-food or 'standard' meals relative to a $9000 \mathrm{~kJ}$ Guideline Daily Amount. Nine dietitians also completed the energy estimation task.

Results: Ratings of prospective satiety generally aligned with the actual size of the meals and indicated that consumers perceived all meals to provide an adequate amount of food, although this differed by gender. The magnitude of the error in energy estimation by consumers was three to ten times that of the dietitians. In both males and females, the average error in energy estimation for the fast-food meals (females: mean 3911 (sD 1998) kJ; males: mean 3382 (sD 1957) kJ) was significantly $(P<0 \cdot 001)$ larger than for the standard meals (females: mean 2607 (SD 1623) kJ; males: mean 2754 (SD 1652) kJ). In women, error in energy estimation for fast-food items predicted actual energy intake from fast-food items $(\beta=0 \cdot 16, P<0 \cdot 01)$.

Conclusions: Knowledge of the energy content of standard and fast-food meals in fast-food consumers in Australia is poor. Awareness of dietary energy should be a focus of health promotion if nutrition information, in its current format, is going to alter behaviour.
\end{abstract}

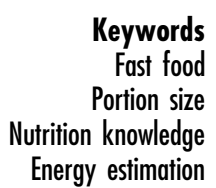

Fast food

Portion size

Energy estimation
Numerous studies have demonstrated that larger portion size results in increased energy intake ${ }^{(1-5)}$. This may, over time, lead to weight gain and obesity. Portion size that is larger than optimal is therefore considered to be an important contributor to overeating and subsequent weight gain ${ }^{(6)}$. The incentive to overeat is also associated with a strong drive for 'value for money' and, consequently, large portions remain appealing to some consumers despite potential health outcomes ${ }^{(7)}$.

Portion size information has many components. In order to use portion size to control intake, consumers need to be aware of the energy density of the foods they are eating and how 'large' these servings are relative to their daily intake. The cognitive processes to achieve this require that individuals are readily able to locate appropriate nutritional information, understand their daily energy requirements, and integrate this information in a way that allows them to manage their intake for the remainder of the day. Previous research supports the suggestion that most people are unable to accomplish these processes ${ }^{(8-10)}$.

Choosing foods that meet serving size recommendations has proven a challenge for nutrition students ${ }^{(8)}$. In another study, $75 \%$ of doctors and nurses were unable to estimate the energy content of food dishes to the closest $250 \mathrm{kcal}^{(9)}(1 \mathrm{kcal}=4 \cdot 184 \mathrm{~kJ})$. Krukowski et al. ${ }^{(10)}$ asked college students and community members to indicate the number of calories they should consume in a day. Using a 'liberal definition', the authors considered any value between 1500 and 2500 correct. Only 67\% of people were able to identify their energy requirements. Combined, these studies indicate that consumers may not possess the skills they require to interpret portion size and use this to moderate consumption. 
Nutrition involvement and/or knowledge may be associated with consumers' ability to interpret and utilise nutrition information. Dieters, who need to be aware of energy content in order to monitor their intake and to meet their dietary restrictions, appear to be more accurate in calorie estimation tasks ${ }^{(11)}$ as well as being frequent users of nutrition labels ${ }^{(12)}$. In a study in the UK, members of the public estimated the number of calories in twelve different food items ${ }^{(13)}$. The estimations made by consumers were close to the actual amounts, when averaged, but highly variable. The authors pooled the absolute error in energy estimation for the food items to create a proxy measure of energy knowledge. They reported that the people with lower BMI had better energy knowledge (were more accurate in their estimations) compared with obese people. However, this was found only in people adjudged as of lower socio-economic status. Other research has suggested that demographic characteristics including gender, socio-economic status, employment status and education levels are associated with general nutrition knowledge ${ }^{(14-16)}$. In another study ${ }^{(17)}$ participants were asked to estimate the amount of energy they had consumed in the fast-food meal they had just eaten as they exited the restaurant. Fast-food consumers with high involvement in nutrition chose smaller meals and were more accurate at estimating the calorie content of the items they had consumed.

There is some indication that engagement with nutrition or nutrition knowledge may be associated with the ability to interpret nutrition information and even food choice. There is also evidence suggesting that consumers struggle to utilise nutrition information. The aims of the present study were to: (i) assess Australian consumers' perception of portion size of fast-food items and their ability to estimate energy content; and (ii) evaluate any association between demographic characteristics, knowledge of energy content in fast-food items and the amount of fast-food consumed. Fast foods were the target of the current study because data were obtained from a survey of fast-food consumers, but this focus was also considered advantageous due to consistent portion sizes and the use of fast foods in other portion size studies ${ }^{(17)}$.

\section{Method}

\section{Participants and procedure}

Perception of portion size was measured as part of a general fast-food survey. This survey asked participants to recall and enter the items consumed at their most recent visit to McDonalds, Hungry Jacks (aka Burger King), KFC, Red Rooster or Domino's Pizza. Recall of the fast-food items eaten was followed by thirty-eight questions about the context of consumption, fast-food behaviours (including frequency of consumption), participant demographics (sex, age, highest level of education, self-reported height and weight, annual household income, postcode, occupation, marital status, country born) and portion size (discussed below). In order to complete the fast-food survey, participants needed to be Australian, over the age of 15 years and have consumed fast food from McDonald's, Hungry Jack's, KFC, Domino's Pizza or Red Rooster in the six months prior to the survey. As an incentive, participants were offered the chance to be entered into the draw to win a double pass to the cinema.

Two methods were used to recruit participants to complete the fast-food survey: a computer station placed in a display for a week at an annual agricultural fair in Adelaide, South Australia and an independent website (www.fastfoodstudy.com.au) which was publicised using local mass media and press releases that directed any fast-food consumers from the age of 16 years upwards to visit the website and complete the survey. At the fair, people walking past were asked if they had $15 \mathrm{~min}$ to do a fast-food survey. Within the display, educational staff delivered interactive science activities that occupied children and allowed parents to do the survey with minimal interruption. In both cases, participants responded to the questions and submitted their responses through the computer. The independent website received 419 responses and 116 people completed the survey at the fair. After data screening, $8 \cdot 6 \%$ of the initial sample was deleted due to missing, incomplete or extreme responses.

Although there were some demographic differences between the two samples recruited on education, household income and employment status, there were no systematic differences in the responses to the portion size items. Therefore results from both cohorts were combined. In the light of documented gender differences in the perception of foods ${ }^{(18,19)}$, the final sample was split by participant sex (168 males, 324 females).

\section{Portion size measures}

The portion size tasks included in the fast-food survey required participants to estimate portion size in terms of two metrics: prospective satiety associated with item consumption and energy (kJ). Five fast-food items and two standard meals were rated. The fast-food items represented 'typical' burger (i.e. McDonald's Big Mac: alone, with medium fries and in a medium meal), chicken (quarter of a chicken and chips) and pizza (four slices of a Hawaiian pizza) meals. The descriptions of the two standard meals used common Australian food items so they were easily recognisable to participants. Each meal was designed to represent either a 'large' meal (T-bone steak with vegetables and sauce) or a 'light' meal (ham sandwich with mayonnaise).

The authors developed the prospective satiety measure to assess how much food each of the meals described represented. Participants were asked to rate the seven meals using a Likert-type scale from 1 ('not enough food') to 7 ('too much food'). 


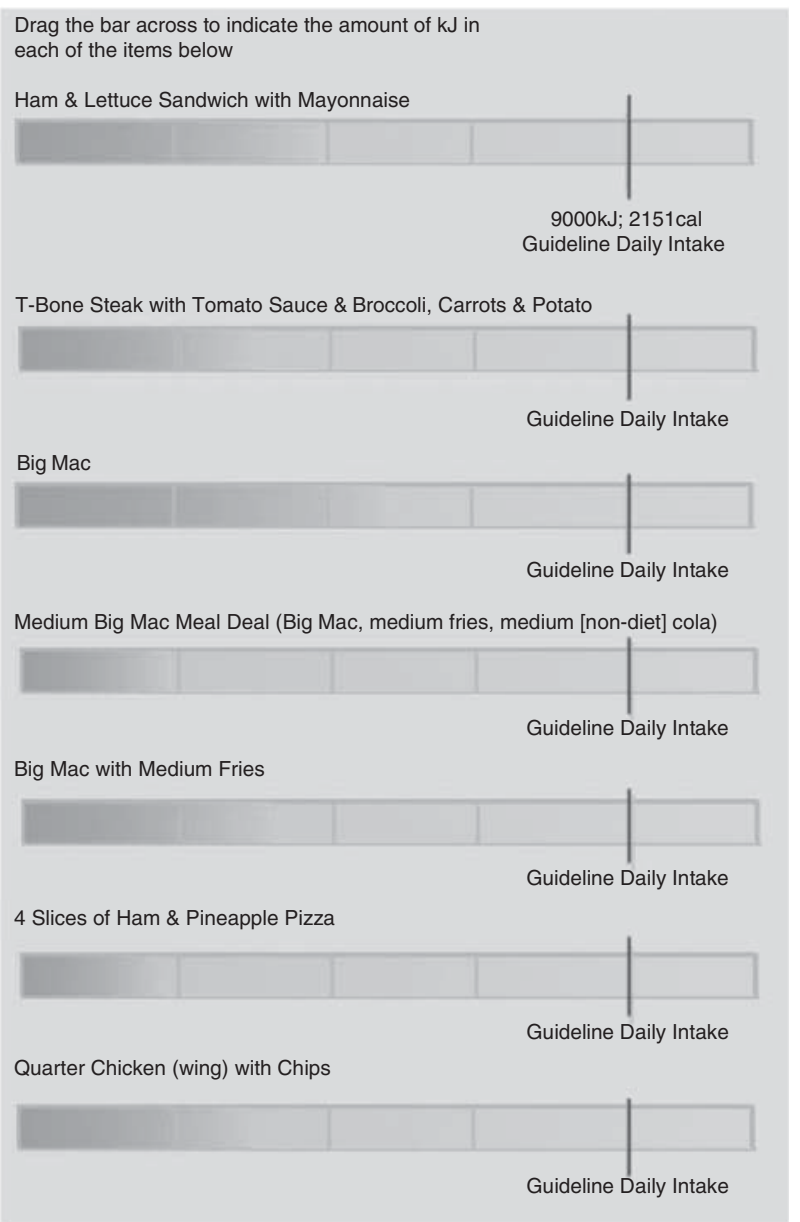

Fig. 1 Screenshot of the kJ estimation task completed by participants

Rather than being asked to estimate an exact value for energy content, as has been done in previous studies $^{(10,11,13,17)}$, participants in the current study entered their energy estimations using a Guideline Daily Amount (GDA) format which consisted of a visual scale showing a $9000 \mathrm{~kJ}$ GDA. This scale had 48 points; the minimum value that could be indicated was $225 \mathrm{~kJ}$ and the maximum was $10800 \mathrm{~kJ}$ (120\% of the daily intake; Fig. 1). Prior to the energy estimation task, participants were told that a 'normal' meal could be conceived of as roughly a third of the daily energy intake. The GDA format was used because it has been rated as valuable and useful in the interpretation of nutrition information by UK consumers ${ }^{(20)}$ and GDA are currently used by big manufacturers (including Kellogg's and McDonalds) for front-of-pack labelling in Australia.

In order to obtain a benchmark of the difficulty of the task, a small sample of dietitians also completed the energy estimation task and provided feedback on its difficulty using a set of six categorical items: $1=$ impossible, $2=$ very difficult, $3=$ difficult, $4=$ reasonable, $5=$ easy, $6=$ very easy. Nine registered and/or practising dietitians completed the questions. All of the dietitians categorised the task as varying in difficulty between reasonable and very easy to complete. When judging difficulty for the general population, two dietitians described the energy estimation task as reasonable difficulty and the remainder considered the task to be difficult to very difficult.

\section{Data analysis}

The energy content of the fast-food items that participants consumed (recalled during the general fast-food survey) was calculated using manufacturer's nutritional information. For the calculation of accuracy in the energy estimation task, the actual kJ content of fast-food items listed in the energy estimation task was gathered from company websites of McDonald's ${ }^{(21)}$, Red Rooster ${ }^{(22)}$ and Domino's Pizza $^{(23)}$ in mid-2006. These brands were used to represent energy content as they are among the most popular quick-service restaurants in Australia ${ }^{(24)}$. Energy information for the standard meals was calculated in FoodWorks 2006 software (Xyris Software (Australia) Pty Ltd, Highgate Hill, Australia) using 'standard' serving sizes.

After reporting their height and weight, participants could indicate if they were confident in the values they had provided. A total of $17 \cdot 3 \%$ of the final sample were not confident in their height and/or weight and were excluded from the calculation of BMI. The subset of participants who were not confident in their BMI values were more likely to be unmarried, unemployed, younger and of lower income than the confident group. Finally, relative socio-economic disadvantage was calculated using the participant's postcode ${ }^{(25,26)}$. Eight responses did not include a valid postcode and were treated as missing.

All resulting data were analysed using the SPSS statistical software package version 16 (SPSS Inc., Chicago, IL, USA) using $t$ tests, multivariate analysis of covariance (MANCOVA) and multiple regression.

\section{Results}

The final sample had a high proportion of female respondents and people in the highest income category (Table 1). There were significant gender differences for age $(t(490)=4 \cdot 29, P<0 \cdot 001)$, monthly fast-food patronage $(t(490)=3.99, \quad P<0 \cdot 001)$, relative socio-economic disadvantage $(t(482)=-2 \cdot 55, P=0 \cdot 01)$, BMI $(t(405)=3 \cdot 64$, $P<0 \cdot 001)$, level of education $\left(\chi^{2}=15.20, P=0 \cdot 004\right)$ and employment status of respondents $\left(\chi^{2}=29 \cdot 64, P<0 \cdot 001\right)$.

\section{Prospective estimation of satiety}

MANCOVA controlling for age, relative socio-economic disadvantage, level of education, income, being full-time employed or married revealed that, in comparison to females, males rated the items as significantly less satiating $(F(7,470)=9 \cdot 29, P<0 \cdot 001)$. The effect of gender remained significant after the inclusion of BMI in the subsample who were confident in their heights and weights $(F(7,386)=$ 9.37, $P<0 \cdot 001)$, suggesting that ratings differed according 
to participant gender and not other demographic differences between males and females.

Both sexes ranked the amount of food for each item roughly in accordance with its weight. The exception was the Medium Big Mac Meal, which weighed slightly less than the pizza and chicken meals, but was rated as providing the second most food - most likely because of the addition of a beverage (Table 2).

Table 1 Demographic characteristics of the males ( $n$ 168) and females ( $n$ 324) in the sample

\begin{tabular}{|c|c|c|}
\hline Variable & Males & Females \\
\hline \multicolumn{3}{|l|}{ Age (years) } \\
\hline Mean & $34 \cdot 21$ & $29 \cdot 51$ \\
\hline SD & $13 \cdot 26$ & $10 \cdot 50$ \\
\hline \multicolumn{3}{|l|}{ BMI $\left(\mathrm{kg} / \mathrm{m}^{2}\right)^{*}$} \\
\hline Mean & $26 \cdot 65$ & $24 \cdot 84$ \\
\hline SD & $4 \cdot 44$ & $5 \cdot 03$ \\
\hline \multicolumn{3}{|l|}{ Monthly fast-food patronage } \\
\hline Mean & $4 \cdot 13$ & $2 \cdot 73$ \\
\hline $\mathrm{SD}$ & $4 \cdot 38$ & $3 \cdot 26$ \\
\hline \multirow{2}{*}{\multicolumn{3}{|c|}{ Household income (\%) }} \\
\hline & & \\
\hline$\$ A U 20000$ and below & $7 \cdot 7$ & $10 \cdot 2$ \\
\hline$\$ A U 20001-40000$ & $8 \cdot 3$ & $13 \cdot 0$ \\
\hline \$AU $40001-60000$ & $24 \cdot 4$ & $26 \cdot 2$ \\
\hline \$AU 60000 and above & $59 \cdot 5$ & $50 \cdot 6$ \\
\hline \multicolumn{3}{|l|}{ Marital status (\%) } \\
\hline Married/living with partner & $54 \cdot 2$ & $46 \cdot 9$ \\
\hline Never married & $41 \cdot \overline{7}$ & $46 \cdot 9$ \\
\hline Other & $4 \cdot 1$ & $6 \cdot 2$ \\
\hline \multicolumn{3}{|l|}{ Highest level of education (\%) } \\
\hline Bachelor & $45 \cdot 8$ & $57 \cdot 4$ \\
\hline Trade/certificate & $28 \cdot 6$ & $19 \cdot 4$ \\
\hline Secondary school & $23 \cdot 8$ & $21 \cdot 3$ \\
\hline Below secondary school & $1 \cdot 8$ & 1.9 \\
\hline \multicolumn{3}{|l|}{ Employment status (\%) } \\
\hline Full-time & $65 \cdot 5$ & $47 \cdot 2$ \\
\hline Student & $12 \cdot 5$ & $27 \cdot 2$ \\
\hline Part-time/casual & $11 \cdot 9$ & $17 \cdot \overline{6}$ \\
\hline Other & $5 \cdot 9$ & $6 \cdot 8$ \\
\hline Unemployed & $4 \cdot 2$ & $1 \cdot 2$ \\
\hline Australian born (\%) & $84 \cdot 5$ & $80 \cdot 2$ \\
\hline
\end{tabular}

*BMI was calculated only for people confident in self-reported heights and weights (145 males, 262 females).

tParticipant's residential postcode was used to calculate the Index of Socioeconomic Disadvantage (IRSD). This index gives an indication of socioeconomic disadvantage according to postal zones throughout Australia. Scores range from 200 to 1200 with lower scores indicating more socioeconomic disadvantage ${ }^{(25)}$
Males did not perceive the Big Mac to provide any more food than the ham sandwich $(t(167)=-0.54$, $P=0 \cdot 59)$, while females did $(t(323)=-5 \cdot 11, P<0 \cdot 001)$. Males rated the T-bone meal as more food than all of the fast-food meals described. Females saw the T-bone meal as more food than the fast-food items with the exception of the Medium Big Mac Meal (Table 3).

\section{Energy estimation}

For the energy estimation task, MANCOVA revealed gender differences when controlling for other demographic variables $(F(7,470)=1 \cdot 45, P<0 \cdot 001)$ and when BMI was included $(F(7,386)=3 \cdot 86, P<0 \cdot 001)$.

The estimations that the dietitians gave were within $10 \%$ of the actual values. On average, males and females in the lay sample estimated large values for all food items (both fast-food and standard items). All participants estimated that the Big Mac Meal contributed a high portion of the daily amount, with females and males indicating that the meal accounted for over $90 \%$ and $80 \%$ of the GDA, respectively (Table 4). Energy content of items was more frequently overestimated than underestimated by both males and females in the lay sample.

The absolute error in energy estimation was calculated by subtracting the actual $\mathrm{kJ}$ content from that estimated by the participants. Absolute error was used due to the small number of underestimations and to focus analyses on the magnitude of error in energy estimation. Average absolute errors made by males and females were three to ten times the magnitude of those made by dietitians (Table 5).

The average absolute error in estimation (herein referred to as 'energy error') for the fast-food items (females: mean 3911 (sD 1998) kJ; males: mean 3382 (sD 1957) kJ) was significantly larger than the average energy error for the standard food items (females: mean 2607 (sD 1623) kJ; males: mean 2754 (sD 1652) kJ) across sexes (both $P<0 \cdot 001$ ). In the dietitian sample, there was no significant difference $(P=0 \cdot 249)$ in the error for fast-food items (mean 616 (SD 263) $\mathrm{kJ}$ ) compared with the energy error for the standard food items (mean 482 (SD 286) kJ). However, this result was likely due to lack of statistical power, given Cohen's $d$

Table 2 Ratings* of prospective satiety in the fast-food and standard food meals for males and females

\begin{tabular}{|c|c|c|c|c|}
\hline & \multicolumn{2}{|c|}{ Malest (n 168) } & \multicolumn{2}{|c|}{ Femalest (n 324) } \\
\hline & Mean & SD & Mean & $\mathrm{SD}$ \\
\hline Ham sandwich (147g) & $3 \cdot 13$ & 0.90 & $3 \cdot 59$ & $1 \cdot 18$ \\
\hline Big Mac $(201 \mathrm{~g})$ & $3 \cdot 21$ & $1 \cdot 38$ & 3.97 & 1.50 \\
\hline Big Mac and medium fries $(299 \mathrm{~g})$ & $3 \cdot 98$ & $1 \cdot 29$ & $4 \cdot 84$ & $1 \cdot 39$ \\
\hline Medium Big Mac Meal $(299 \mathrm{~g})$ & $4 \cdot 46$ & $1 \cdot 35$ & $5 \cdot 29$ & $1 \cdot 50$ \\
\hline Four slices of Hawaiian pizza $(316 \mathrm{~g})$ & $4 \cdot 18$ & $1 \cdot 49$ & $5 \cdot 02$ & $1 \cdot 40$ \\
\hline Quarter chicken and chips (441 g) & $4 \cdot 30$ & $1 \cdot 16$ & $5 \cdot 02$ & $1 \cdot 31$ \\
\hline T-bone meal $(447 \mathrm{~g})$ & $4 \cdot 82$ & 1.07 & $5 \cdot 29$ & $1 \cdot 16$ \\
\hline
\end{tabular}

*Each food was rated from $1=$ not enough food to $7=$ too much food.

tThere were significant sex differences for the ratings of all items $(P<0.001)$. 
Table 3 Comparison of ratings of prospective satiety for the large standard meal relative to the fast-food meals

\begin{tabular}{|c|c|c|c|c|}
\hline & \multicolumn{2}{|c|}{ Males $(\mathrm{df}=167)$} & \multicolumn{2}{|c|}{ Females $(\mathrm{df}=323)$} \\
\hline & $t$ & $P$ & $t$ & $P$ \\
\hline T-bone meal $(447 \mathrm{~g})$ & Ref. & Ref. & Ref. & Ref. \\
\hline Big Mac and medium fries $(299 \mathrm{~g})$ & $7 \cdot 12$ & $<0.001$ & $5 \cdot 50$ & $<0.001$ \\
\hline Medium Big Mac Meal (299g) & 3.03 & 0.003 & $-0 \cdot 11$ & 0.910 \\
\hline Four slices of Hawaiian pizza $(316 \mathrm{~g})$ & $5 \cdot 46$ & $<0.001$ & 3.09 & 0.002 \\
\hline Quarter chicken and chips ( $441 \mathrm{~g})$ & $4 \cdot 08$ & $<0.001$ & $3 \cdot 24$ & 0.001 \\
\hline
\end{tabular}

Ref., reference.

Table 4 Estimated kilojoules (kJ) of food items (Actual) and this value as a percentage of the Guideline Daily Amount (\%GDA) with estimations for dietitians, males and females

\begin{tabular}{|c|c|c|c|c|c|c|c|c|c|c|c|}
\hline & \multicolumn{2}{|c|}{ Actual } & \multicolumn{3}{|c|}{ Dietitians* } & \multicolumn{3}{|c|}{ Males* } & \multicolumn{3}{|c|}{ Females* } \\
\hline & $\mathrm{kJ}$ & $\% G D A$ & Mean & SD & $\% G D A$ & Mean & SD & $\% G D A$ & Mean & SD & $\% G D A$ \\
\hline Ham sandwicht & 1179 & $13 \cdot 1$ & 1252 & 493 & $13 \cdot 9$ & 3630 & 1854 & $40 \cdot 3$ & 3565 & 1857 & $39 \cdot 6$ \\
\hline Big Mac & 2010 & $22 \cdot 3$ & 2304 & 387 & $25 \cdot 6$ & 5701 & 2553 & $63 \cdot 3$ & 6211 & 2544 & $69 \cdot 0$ \\
\hline T-bone mealt & 2157 & $24 \cdot 0$ & 2404 & 676 & $26 \cdot 7$ & 5136 & 2003 & $57 \cdot 1$ & 4976 & 1858 & $55 \cdot 3$ \\
\hline Four slices of Hawaiian pizza & 2928 & $32 \cdot 5$ & 3731 & 1009 & $41 \cdot 5$ & 6353 & 2167 & $70 \cdot 6$ & 7069 & 2277 & $78 \cdot 5$ \\
\hline Big Mac and medium fries & 3270 & $36 \cdot 3$ & 3531 & 374 & $39 \cdot 2$ & 6507 & 2347 & $72 \cdot 3$ & 7133 & 2266 & $79 \cdot 3$ \\
\hline Quarter chicken and chips & 3741 & $41 \cdot 6$ & 3205 & 907 & $35 \cdot 6$ & 6217 & 2238 & $69 \cdot 1$ & 6586 & 2228 & $73 \cdot 2$ \\
\hline Medium Big Mac Meal & 3866 & $43 \cdot 0$ & 4282 & 465 & $47 \cdot 6$ & 7309 & 2432 & $81 \cdot 2$ & 8174 & 2299 & $90 \cdot 8$ \\
\hline
\end{tabular}

*All values were significantly different between the population samples (both males and females) and the dietitians at the $P<0 \cdot 001$ level.

tAs estimated by FoodWorks 2006 software (Xyris Software (Australia) Pty Ltd, Highgate Hill, Australia).

Table 5 Average error (in $\mathrm{kJ}$ ) and the percentage of the sample underestimating the $\mathrm{kJ}$ content of the food items rated in the energy estimation task (\%under) for dietitians, males and female sample

\begin{tabular}{|c|c|c|c|c|c|c|c|c|c|}
\hline & \multicolumn{3}{|c|}{ Dietitians } & \multicolumn{3}{|c|}{ Males } & \multicolumn{3}{|c|}{ Females } \\
\hline & Mean & SD & \%under & Mean & SD & \%under & Mean & SD & \%under \\
\hline Ham sandwich & 381 & 292 & $55 \cdot 6$ & 2467 & 1847 & $3 \cdot 0$ & 2400 & 1820 & $5 \cdot 9$ \\
\hline Big Mac & 386 & 281 & $22 \cdot 2$ & 3747 & 2507 & $3 \cdot 6$ & 4191 & 2513 & $1 \cdot 2$ \\
\hline T-bone meal & 583 & 378 & $33 \cdot 3$ & 3040 & 1934 & $6 \cdot 6$ & 2814 & 1815 & $1 \cdot 2$ \\
\hline Four slices of Hawaiian pizza & 952 & 850 & $22 \cdot 2$ & 3504 & 2074 & $3 \cdot 6$ & 4133 & 2236 & $2 \cdot 5$ \\
\hline Big Mac and medium fries & 362 & 262 & $22 \cdot 2$ & 3350 & 2218 & $6 \cdot 0$ & 3907 & 2162 & $3 \cdot 4$ \\
\hline Quarter chicken and chips & 846 & 583 & $66 \cdot 7$ & 2689 & 1992 & $14 \cdot 3$ & 2972 & 2054 & $10 \cdot 8$ \\
\hline Medium Big Mac Meal & 532 & 304 & $22 \cdot 2$ & 3622 & 2181 & $8 \cdot 9$ & 4353 & 2195 & $2 \cdot 5$ \\
\hline
\end{tabular}

for the comparison between the dietitians' ratings approximated $0 \cdot 5$, indicating that the mean difference corresponded to a medium effect size $\mathrm{e}^{(27)}$.

\section{Relationship between energy error, demographics and behaviour}

The associations between participant demographics, energy error for fast-food items (as calculated above) and the average energy consumed from fast foods at the previous fast-food eating occasion (recalled in the fastfood survey) were determined by multiple linear regressions. In the first regression, demographic characteristics (participant age, working full-time, household income, level of education, relative socio-economic disadvantage and frequency of fast-food consumption) were entered as predictors for energy error for the fast-food items (calculated from the energy estimation task). In the second regression, energy error and the participant demographics were used as predictors for the amount of fast-food eaten (in $\mathrm{kJ}$ ) at the participants' previous visit to a fast-food restaurant. According to the requirement of twenty participants per predictor for regression ${ }^{(28)}$, the model including seven predictors was adequately powered to detect effects in both the male and female samples considered.

None of the demographic characteristics were significant predictors of energy error, and overall the models were not significant for males or females. The model predicting energy intake from fast-food items in males also failed to reach significance but was significant in females $(F(7,310)=2 \cdot 67, P=0 \cdot 01)$. The model explained $3 \cdot 5 \%$ of the total variance (based on adjusted $R^{2}$ ), with energy error as the single significant predictor of energy intake. The $\beta$ value for energy error was $0 \cdot 16$, indicating that greater error in energy estimation was associated with slightly greater 
energy intake from fast-food items. The unstandardised value indicated that each $1000 \mathrm{~kJ}$ increment in energy error was associated with a 127 (SE 45) $\mathrm{kJ}$ increase in intake.

Given the potential importance of BMI in predicting general nutrition knowledge ${ }^{(13)}$, the models predicting energy error were re-run, including BMI, for the subset of people who indicated confidence in their weights and heights (145 males, 262 females). Despite the inclusion of BMI, the models predicting energy error were not significant in both males and females.

\section{Discussion}

The study presented in the current paper aimed to explore consumers' perceptions of the portion size of fast-food and standard meals as well as assessing the relationship between error in energy estimation and actual intake of fast foods. When asked to indicate prospective satiety of multiple meals, most consumers thought that the food items described provided an adequate volume of food. Fast-food meals were rated as containing a smaller volume of food than the standard large meal, with the exception of women's ratings of the Big Mac. In contrast, when estimating the energy content of the meal, many consumers indicated that food items contained enough energy to account for a large portion of the GDA. Generally, consumers' errors in energy estimation were large and none of the demographic characteristics considered predicted the magnitude of this error. In women there was some evidence that the degree to which they incorrectly estimated the energy content of the fast-food items could predict the amount of fast food they consumed at their last eating occasion.

The lay samples indicated that fast foods provided high amounts of energy. This was evident in their rating of the Medium Big Mac Meal. Men and women rated the amount of energy in this meal almost equivalent to the entire GDA. Admittedly, not all burger meals are the same. The equivalent burger meal from Hungry Jacks accounts for approximately $25 \%$ more energy than the Big Mac meal from McDonalds ${ }^{(24)}$, but this still only represents just over half of the $9000 \mathrm{~kJ}$ GDA used. If consumers believe that a fast-food meal accounts for almost a whole day's energy requirements, it would be useful to investigate how they would, and actually do, cope with this perceived excessive intake. Consumers may attempt to counterbalance this intake with restricted food intake throughout the day. However, the perception of excessiveness could also result in increased consumption throughout the day if dietary disinhibition occurs ${ }^{(29)}$.

It was interesting that the energy estimations were more often overestimations than underestimations, as this contradicts previous research ${ }^{(17)}$. The health halo effect describes the tendency to underestimate the energy content of food claimed to be healthy, such as Subway ${ }^{(30)}$. The standard food items were overestimated along with the fast-food items. Yet errors in energy estimation for fast-food meals were larger than those for the standard meals, suggesting that perhaps the perceived 'unhealthiness' of these items increased the errors made. However, here the average energy content of the fast-food items was greater than that of the standard food items, meaning that the greater error may also be a function of different meal sizes ${ }^{(17)}$.

Nestle $^{(31)}$ discusses a 'virtual absence of intuitive understanding that larger portions contribute more calories' (p. 40). As items were added to the Big Mac, both males and females increased their energy estimations suggesting some understanding that more food (and even drink) can provide more energy. Obviously energy estimation was further complicated by energy density of fastfood items in the current study. Here, the T-bone meal represented the greatest volume of food and yet only an intermediate amount of energy. It appeared that consumers had some understanding of this since, on average, the steak meal was rated as having less energy than all of the fast-food items. It is unclear whether the perception that fast food has more energy than standard meals is related to the perception of fast foods as unhealthy or an actual understanding of energy density. Data indicating that $75 \%$ of an Australian sample was unaware that fat is energy dense ${ }^{(14)}$ suggest that the latter is unlikely.

Despite some adjustment for portion size, consumers made gross errors in energy estimation. If the pooled error in estimation is treated as a proxy measure for energy awareness or knowledge ${ }^{(13)}$, the magnitude of error in the current sample indicates that they had poor awareness of the energy content of food items. Although this may also reflect the complexity of the task for consumers (predicted by the dietitian sample who indicated it would be difficult for the general population), estimating the impact of a meal on a day's energy requirements is no more challenging than the processes involved in interpreting and using the details displayed on nutrition information panels in Australia. If nutrition information remains in its current format, consumers need further education or strategies to interpret and utilise energy information and balance energy intake.

The results for the female sample suggested that accuracy at estimating energy content may be related to behaviour. Women who were better at the energy estimation task (i.e. had a lower absolute energy estimation error) also consumed less energy at their previous fast-food consumption occasion, which suggests that a better understanding of energy content may be associated with intake. Error in estimation predicted intake more strongly than other demographic factors, such as age, but accounted for only a small amount of the total variance for the amount of fast food consumed.

The ability to estimate energy may be associated with behaviour in women and not men because of a high involvement in health and dieting. Certainly, this would be consistent with results from a large-scale multi-country study in which women were reported to be more likely to be dieting than men ${ }^{(32)}$ and Australian census data which 
reveal that there are almost 3.5 times more women majoring in a health field than men ${ }^{(33)}$. Gender differences in concern about food require more research especially if they have the potential to moderate effects on dietary behaviour.

Public education about the energy content of food in relation to daily requirements may improve consumers' ability to interpret and utilise this information. General nutrition knowledge has been associated with dietary behaviours such as lower consumption of foods away from home ${ }^{(34)}$. There is evidence that calorie counting is a skill that can be taught ${ }^{(35)}$. Indeed, estimating the energy content of foods is a skill that many dieters adapt and put in practice. Increasing involvement with nutrition together with selfefficacy may also be a promising way of increasing the effect of nutrition knowledge on behaviour ${ }^{(36)}$. An alternative (and potentially cost-effective) approach to education is the provision of intelligent multimedia tools that utilise 'just-in-time' prompting strategies and self-monitoring programmes ${ }^{(37,38)}$. Yet another solution may be to implement policies that change the way that nutrition information is communicated, which may also enable individuals to monitor and moderate their energy intake more effectively.

The above approach has been recently adopted in the USA where calorie information will be required to be displayed at the point of purchase in food establishments ${ }^{(39)}$. The efficacy of a labelling and informational approach for energy moderation is supported by some ${ }^{(40,41)}$ but not all studies $^{(10,42,43)}$. In the specific case of fast food, less than $5 \%$ of consumers reported seeing nutrition information in fastfood restaurants in New York City (where labelling has been compulsory since 2007) but, when they did, they consumed fewer calories ${ }^{(44)}$. Therefore, making energy content information prominent for fast-food items may positively alter behaviour in a limited number of cases. However, the possibility that consumers' drive for value for money may also undermine this tactic needs consideration, with companies already advertising items with calories per dollar ${ }^{(45)}$. Future studies are needed to clarify the efficacy of providing energy contents at a population level and to determine if environmental cues can increase awareness of dietary energy and/or alter behaviour.

The current findings are subject to methodological limitations. It is possible that the survey attracted certain types of people such as those with higher involvement with food and nutrition. The sample was slightly homogeneous lacking a strong representation of men or people of low socioeconomic status. This may have may have limited the ability to replicate previous findings of an association between energy knowledge and $\mathrm{BMI}^{(13)}$. The presentation of the tasks could also be improved in the future. Previous studies have asked participants to rate expected satiety by presenting photographs which could be incorporated into a new version of the task, particularly when assessing nonstandardised meals (i.e. chicken and chips) ${ }^{(46)}$.

The present study has provided unique data on the way in which Australian consumers perceive the size of fast-food items. Improved understanding of dietary energy is required if consumers are to balance their energy intake with their requirements. If future portion size interventions are to be successful, more data on the perception of portion size and consumer knowledge are needed to determine the most effective strategies for behaviour change.

\section{Acknowledgements}

The study was funded by a Linkage Grant from the Australian Research Council. The industry partners in this grant are the South Australian Health Department and McDonald's Australia. Neither industry partner had a role in the designing, analysing or interpreting the study or in the drafting, review or approval of the manuscript. G.W. is a member of the McDonald's Global Advisory Council on Balanced, Active Lifestyles, on which he provides advice about nutrition and contributes to their balanced active lifestyles policy and programme. There were no further conflicts of interest. E.B. conceived the study and conducted the research and analysis for the study, which formed part of her PhD thesis. C.W., P.M. and G.W. provided academic supervision and support for the work. E.B. prepared the manuscript. All authors contributed to, read and approved the final manuscript.

\section{References}

1. Diliberti N, Bordi PL, Conklin MT et al. (2004) Increased portion size leads to increased energy intake in a restaurant meal. Obes Res 12, 562-568.

2. Ledikwe JH, Ello-Martin JA \& Rolls BJ (2005) Portion sizes and the obesity epidemic. J Nutr 135, 905-909.

3. Rolls BJ, Roe LS, Kral TVE et al. (2004) Increasing the portion size of a packaged snack increases energy intake in men and women. Appetite 42, 63-69.

4. Wansink B, Painter JE \& North J (2005) Bottomless bowls: why visual cues of portion size may influence intake. Obes Res 13, 93-100.

5. Wansink B \& Park SB (2001) At the movies: how external cues and perceived taste impact consumption volume. Food Qual Prefer 12, 69-74.

6. Herman CP, Fitzgerald NE \& Polivy J (2003) The influence of social norms on hunger ratings and eating. Appetite $\mathbf{4 1}$, $15-20$.

7. Vermeer WM, Steenhuis IHM \& Seidell JC (2009) From the point-of-purchase perspective: a qualitative study of the feasibility of interventions aimed at portion-size. Health Policy 90, 73-80.

8. Young LR \& Nestle M (1998) Variation in perceptions of a 'medium' food portion: implications for dietary guidance. J Am Diet Assoc 98, 458-459.

9. Ashley BD, Davidson B, Wilkins J et al. (2004) Counting calories - how good are health care professionals? Nutrition 20, 817-820.

10. Krukowski RA, Harvey-Berino J, Kolodinsky J et al. (2006) Consumers may not use or understand calorie labeling in restaurants. J Am Diet Assoc 106, 917-920.

11. Carels RA, Konrad K \& Harper J (2007) Individual differences in food perceptions and calorie estimation: an examination of dieting status, weight, and gender. Appetite 49, 450-458. 
12. Neuhouser ML, Kristal AR \& Patterson RE (1999) Use of food nutrition labels is associated with lower fat intake. J Am Diet Assoc 99, 45-53.

13. Speakman JR, Walker H, Walker L et al. (2005) Associations between BMI, social strata and the estimated energy content of foods. Int J Obes (Lond) 29, 1281-1288.

14. Hendrie GA, Cox DN \& Coveney J (2008) Validation of the general nutrition knowledge questionnaire in an Australian community sample. Nutr Diet 65, 72-77.

15. O'Brien G \& Davies M (2007) Nutrition knowledge and body mass index. Health Educ Res 22, 571-575.

16. Parmenter K, Waller J \& Wardle J (2000) Demographic variation in nutrition knowledge in England. Health Educ Res 15, 163-174.

17. Chandon P \& Wansink B (2007) Is obesity caused by calorie underestimation? A psychophysical model of meal size estimation. J Mark Res 44, 84-99.

18. Oakes ME \& Slotterback CS (2001) Gender differences in perceptions of the healthiness of foods. Psychol Health 16, $57-65$.

19. Oakes ME \& Slotterback CS (2001) What's in a name? A comparison of men's and women's judgements about food names and their nutrient contents. Appetite 36, 29-40.

20. Sadler M (1998) Making nutrition labelling more accessible for consumers. BNF Nutr Bull 23, August issue, 142-149.

21. McDonald's Corporation (2006) Nutrition information. http:// mcdonalds.com.au/PDFs/Master_Nutrition_Information.pdf (accessed June 2006)

22. Australian Fast Foods Pty Ltd (2006) Red Rooster Nutrition Brochure. http://www.redrooster.com.au/pdfs/Red\%20Rooster \%20Nutritional\%20Brochure.pdf (accessed July 2006).

23. Domino's Pizza Enterprises Ltd (2006) Nutritional information. http://www.dominos.com.au/menu/nutritional (accessed July 2006).

24. Brindal E, Mohr P, Wilson C et al. (2008) Obesity and the affects of choice at a fast food restaurant. Obes Res Clin Pract 2, 111-117.

25. Australian Bureau of Statistics (2008) An Introduction to Socio-Economic Indexes for Areas (SEIFA). Report no. 2039.0. Canberra: The Australian Government.

26. Australian Bureau of Statistics (2008) Socio-Economic Indexes for Areas (SEIFA) - Technical Paper. Report no. 2039.0.55.001. Canberra: The Australian Government.

27. Cohen J (1988) Statistical Power Analysis for the Behavioral Sciences, 2nd ed. Hillsdale, NJ: Lawrence Erlbaum Associates.

28. Tabachnick B \& Fidell L (1989) Using Multivariate Statistics, 2nd ed. New York: Harper Collins.

29. Herman CP \& Polivy J (2007) Norm-violation, normadherence, and overeating. Coll Antropol 31, 55-62.

30. Wansink B \& Chandon P (2006) Health halos: how nutrition claims influence food consumption for overweight and normal weight people. FASEB J 20, A1008.
31. Nestle M (2003) Increasing portion sizes in American diets: more calories, more obesity. J Am Diet Assoc 103, 39-40.

32. Wardle J, Haase AM, Steptoe A et al. (2004) Gender differences in food choice: the contribution of health beliefs and dieting. Ann Behav Med 27, 107-116.

33. Australian Bureau of Statistics (2007) 2006 Census: Community Profile Series. Report no. 2001.0. Canberra: The Australian Government.

34. Beydoun MA, Powell LM \& Wang YF (2009) Reduced awayfrom-home food expenditure and better nutrition knowledge and belief can improve quality of dietary intake among US adults. Public Health Nutr 12, 369-381.

35. Martin CK, Anton SD, York-Crowe E et al. (2007) Empirical evaluation of the ability to learn a calorie counting system and estimate portion size and food intake. Br J Nutr 98, 439-444.

36. Rimal RN (2000) Closing the knowledge-behavior gap in health promotion: the mediating role of self-efficacy. Health Commun 12, 219-237.

37. Atienza AA, King AC, Oliveira BM et al. (2008) Using handheld computer technologies to improve dietary intake. Am J Prev Med 34, 514-518.

38. Tsai C, Lee G, Raab F et al. (2007) Usability and feasibility of PmEB: a mobile phone application for monitoring real time caloric balance. Mobile Networks Applic 12, 173-184.

39. Bernstein $S$ (2010) Calorie counts to be required on menus nationwide. Los Angeles Times, 23 March; available at http://articles.latimes.com/2010/mar/23/business

40. Cranage D, Conklin MT \& Lambert C (2005) Effect of nutrition information in perceptions of food quality, consumption behavior and purchase intentions. J Foodservice Bus Res 7, 43-61.

41. Gerend MA (2009) Does calorie information promote lower calorie fast food choices among college students? J Adolesc Health 44, 84-86.

42. Harnack LJ, French SA, Oakes JM et al. (2008) Effects of calorie labeling and value size pricing on fast food meal choices: results from an experimental trial. Int J Behav Nutr Phys Act 5, 63.

43. Yamamoto JA, Yamamoto JB, Yamamoto BE et al. (2005) Adolescent fast food and restaurant ordering behavior with and without calorie and fat content menu information. J Adolesc Health 37, 397-402.

44. Bassett MT, Dumanovsky T, Huang C et al. (2008) Purchasing behavior and calorie information at fast-food chains in New York City, 2007. Am J Public Health 98, 1457-1459.

45. Gimein M (2009) The Big Max - Using New York's caloriedisclosure regulation to get the most for your money. The Big Money. http://www.thebigmoney.com/article/judge ments/2009/05/17/big_max (accessed June 2009).

46. de Graaf C, Stafleu A, Staal P et al. (1992) Beliefs about the satiating effect of bread with spread varying in macronutrient content. Appetite 18, 121-128. 